\section{Tabakabhängigkeit und Möglichkeiten der medikamentösen Behandlung}

Zusammenfassung: Die Tabakabhängigkeit ist aufgrund der steigenden Raucherzahlen in allen Industrieländern als wachsende Bedrohung für den Gesundheitszustand der Bevölkerung anzusehen. Dabei ist die mit Alkohol und Heroin vergleichbare abhängigkeitserzeugende Wirkung für den jahrzehntelangen Tabakkonsum verantwortlich. Die Abhängigkeit wird exogen ausgelöst, jedoch kommt aufgrund der bisherigen Daten auch eine genetische Komponente in Betracht, wobei Parallelen zur Alkoholabhängigkeit gegeben sind. Für die Behandlung der Tabakabhängigkeit werden verschiedene verhaltenstherapeutische Verfahren empfohlen, die jedoch vom Raucher weniger akzeptiert werden als die medikamentösen Verfahren, unter denen die vorübergehende Nikotinbehandlung die derzeitig erfolgreichste Methode darstellt. Des Weiteren wird die Behandlung mit Bupropion abgehandelt, die jedoch aufgrund der bisher international beschriebenen unerwünschten Wirkungen eine weniger günstige Nutzen-Risiko-Relation als die zeitweilige Nikotinsubstitution darstellt.

Schlüsselwörter: Tabakabhängigkeit - Nikotin - Nikotinabhängigkeit - Diagnostik - Medikamentöse Therapie Nikotinersatzprodukte - Bupropion

Tobacco Dependence and Possibilities of Smoking Cessation Therapy: Due to increasing smoker numbers in all industrial countries the tobacco dependence can be seen as growing threat for the state of health to the population. The dependence producing effect of nicotine is comparable with those of alcohol and heroin and, furthermore the alkaloid is responsible for decades of tobacco consumption of each subject. Nicotine dependence is triggered exogenously, a genetic component is also considered. On the basis of the present data parallels exist to the alcohol dependence. For smoking cessation various behavior therapy programs are used which, however, are accepted by the smoker to a smaller degree than nicotine replacement therapy (NRT). NRT represents the most successful method at time. Bupropion therapy possesses a less favorable use-risk relation than NRT because of its side-effects.

Suchttherapie 2001; 2: 209-217

(c) Georg Thieme Verlag Stuttgart · New York ISSN 1439-9903
Knut-Olaf Haustein

Institut für Nikotinforschung und Raucherentwöhnung, Erfurt
Key words: Tobacco Dependence - Nicotine - Nicotine Addiction - Diagnostics - Nicotine Replacement Therapy Bupropion

Die Tabakabhängigkeit hat in allen Industrieländern beängstigende Ausmaße angenommen, wobei in Deutschland wie auch in einigen anderen Ländern 97\% der Raucher Zigaretten konsumieren. Nach der Mikrozensusstudie von 1999 rauchen im Erwachsenenalter (hier berücksichtigt das 20. bis 55 . Lebensjahr) zwischen 35 und 40\% der Frauen und 40 und $45 \%$ der Männer Zigaretten [1]. Die Zahl der Abhängigen im Sinne einer Sucht wird in Deutschland auf 6,8 Millionen geschätzt bei einer etwa 3fachen Menge an Rauchern [2]. In Deutschland haben wir derzeit täglich 309 Tote durch den Tabakkonsum zu beklagen, das sind 110000 Tote jährlich [2,3]. Demgegenüber sterben auf Deutschlands Straßen täglich „nur 21 Menschen“. Über Letztere wird in der Öffentlichkeit mehr diskutiert als über die 15 fache Zahl von Tabaktoten. Betrachtet man die Vorverlegung des Einstiegsalters mit dem Rauchbeginn und die rascher zunehmende Anzahl von Raucherinnen im Kindesalter, dann sind hier endlich wirksame Entscheidungen unserer Politiker neben verstärkten ärztlichen Maßnahmen zur Eindämmung dieser katastrophalen Entwicklung angezeigt [4].

\section{Die Tabakabhängigkeit und Typisierung der Sucht}

Nikotin ist als Suchtstoff der Tabakindustrie seit etwa 50 Jahren bekannt. Nikotin stimuliert die Freisetzung von Mediatoren. Insbesondere das dopaminerge System als Belohnungssystem wird durch Nikotin beeinflusst [5]. Im Unterschied zum Alkohol, der „zweiten legalen Droge“ ist Nikotin kaum psychotoxisch wirksam, was u.a. auch bedeutet, dass der selbst stark abhängige Raucher nur geringe soziale Auffälligkeiten zeigt. 17\% der Raucher sind stark abhängig [6], so dass dieser Personenkreis auch nur sehr schwer zu entwöhnen ist - im Allgemeinen nicht ohne ärztliche Hilfe.

Der Fagerström Test for Nicotine Dependence (FTDN) lässt den Schweregrad der Abhängigkeit auf der Basis von 6 Fragen und der Höhe der erreichten Punkte (maximal 10) bewerten, wobei eine starke Abhängigkeit mit einem Punktwert $>7$ einhergeht [6]. Nach dem statistischen Manual der Amerikanischen Psychiatrischen Gesellschaft (DSM-IV [7]) wird eine Nikotinabhängigkeit angenommen, wenn 3 von 7 Beurteilungskriterien erfüllt sind, z.B. Toleranzbildung, Entzugserscheinungen, Rauchen größerer Mengen als eigentlich 
beabsichtigt, Rauchen trotz detaillierten Wissens über die Gesundheitsschäden.

\section{Molekularbiologische Aspekte der Sucht}

Geringe Nikotindosen, vergleichbar mit denen beim Zigarettenrauchen, wirken bei mehrmaliger Applikation ähnlich wie Amphetamin oder Kokain motorisch stimulierend [8], so dass Nikotin als Psychostimulans zu bewerten ist. Die lokomotorisch erregenden und „selbstbelohnenden“ Nikotinwirkungen werden über eine verstärkte Liberation von Dopamin aus dem Nucleus accumbens im hinteren Anteil des mesolimbischen Systems bewirkt [8,9]. Die Mehrsekretion von Dopamin kommt über eine erhöhte Impulsdichte zustande [10,11]. Parallel mit der Stimulation der Dopaminsekretion wird der N-Methyl-D-Aspartat-(NMDA-)Rezeptor stimuliert, der für die Bindung von Glycin und die Potenzierung der Wirkung von Glutamat zuständig ist $[12,13]$. Die verstärkte Dopaminfreisetzung hängt zusammen mit einem Nikotin bevorzugenden Verhalten, welches vom „Lieben“ zum „Verlangen“ umschlägt. Damit ist es sehr wahrscheinlich, dass die Nikotinabhängigkeit über die gleichen Dopamin-Reaktionen abläuft wie die von Amphetamin und Kokain [14]. Da Nikotin aber über mehrere Rezeptoren lang anhaltend wirkt, werden diese bei Langzeitanwendung desensibilisiert [15]. Zusätzlich stimuliert Nikotin die Freisetzung von Noradrenalin aus Anteilen des ventralen Hippocampus über Isoformen des Nikotinrezeptors, die vom Locus coeruleus innerviert und durch ständig anwesendes Nikotin desensibilisiert werden [16].

Die fortwährende Nikotinzufuhr führt im Hippocampus zu einer regionalen Abnahme der 5-HT-Synthese und -Konzentration $[17,18]$. Nikotin vermindert den 5-HT-Überschuss im Hippocampus, was auf anxiolytische Wirkungen des Nikotins hinweisen könnte $[19,20]$. Tatsächlich rauchen depressive Patienten häufiger als Gesunde, was auf eine antidepressive Wirkung des Tabakrauchs schließen lässt [21].

\section{Genetische Aspekte der Tabakabhängigkeit}

Genetische Aspekte scheinen bei der Ausbildung der NikotinAbhängigkeit weniger bedeutsam zu sein als Umwelteinflüsse (Eltern, Schulgruppen, Erziehung, religiöse Aspekte, Haltung der Gesellschaft, Werbung) [22,23]. Dementgegen ist das starke Rauchen oder die Unfähigkeit, das Rauchen abzubrechen, nicht mehr mit Umgebungseinflüssen zu erklären, so dass dafür biologische (medikamentöse Einflüsse, psychiatrische Störungen, Neuroadaptation) und genetische Effekte [24,25] verantwortlich zu machen sind, wie aus der Zwillingsforschung zu erkennen ist, wobei ein Zusammenhang zwischen Persönlichkeitsfaktoren und Rauchverhalten beim monozygoten stärker als bei dizygoten Zwilling hervortrat [25]. Da zahlreiche Einflussfaktoren wirksam werden, stützen sich die Aussagen auf einige Befunde:

1. Das Enzym $\mathrm{CYP}_{2 \mathrm{~A} 6}$ metabolisiert Nikotin zu Cotinin. Träger einer defekten Enzymvariante bauen Nikotin verzögert ab und weisen eine verminderte Nikotinabhängigkeit auf [26]. Defekte Allele sind mit 1-3\% seltener als bisher angenommen (z.B. bei Finnen, Spaniern und Schweden) [27].

2. Im ZNS sind Untergruppen von Dopamin-Rezeptoren $\left(\mathrm{DA}_{1}\right.$ und $\left.\mathrm{DA}_{2}\right)$ nachgewiesen worden. Auch nach Untersuchungen an Patienten mit Lungenkarzinom könnten abweichende Allele des $D_{2}$-Rezeptors eine Rolle für die Ausbildung der Nikotinabhängigkeit spielen [28].

3. Die im Genomfragment $\lambda \mathrm{hD} 2 \mathrm{G} 1$ vorkommenden Allele TaqI-A1 (20\%) und TaqI-A2 sind in der Bevölkerung unterschiedlich verteilt, wobei Alkoholiker zu 50-60\% Träger dieses Allels sind $[29,30]$. Über ein vermehrtes Zusammentreffen von DRD2-A1-Allel und Alkoholikern (45\%) bei Vergleich mit der Allgemeinbevölkerung (25\%) wurde berichtet [29,31]. Diese Form der genetisch bedingten Abhängigkeit scheint auch für Raucher zuzutreffen $[8,9,28]$ : Das Vorkommen war gegenüber der Normalbevölkerung erhöht (48,7 vs. 25,9\%) [32,33]. Träger dieses A1-Allels beginnen früher zu rauchen und können auch nur kurze Zeiten abstinent bleiben [33]. Es wird angenommen, dass das A1-Allel mit einer herabgesetzten Aktivität der D2-Rezeptoren bei geringerer Rezeptorendichte, aber intakter Funktion einhergeht [32].

4. In Verbindung mit dem am Chromosom 11 lokalisierten $\mathrm{D}_{2}$-Dopaminrezeptor $\left(\mathrm{DRD}_{2}\right)$ existiert ein veränderter Dopamintransporter (SLC6A3): Patienten mit dem SLC6A39-Genotyp in Verbindung mit dem $\mathrm{DRD}_{2}-\mathrm{A}_{2}$-Genotyp waren seltener Raucher [34], Träger des SLC6A3-Gens hingegen häufiger Raucher und nikotinabhängig.

5. Zwischen der Alkohol- und der Nikotinabhängigkeit bestehen genetische Gemeinsamkeiten [35], wobei die Vererbbarkeit für die Nikotinabhängigkeit bei $60,3 \%$ und die für die Alkoholabhängigkeit bei $55,1 \%$ lag. Die fetale Alkoholexposition führt nach neueren Untersuchungen zu einem erhöhten Risiko für das spätere Aufkommen einer Abhängigkeit für Nikotin, Alkohol und verschiedene Drogen [36].

\section{Entzugserscheinungen}

Entzugserscheinungen können bei einer starken Abhängigkeit von Nikotin mit einem täglichen Zigarettenkonsum von > 20 Zigaretten auftreten, wobei die erste Zigarette morgens unmittelbar nach dem Aufwachen geraucht wird oder aber der Raucher auch nachts munter wird und dann 1-2 Zigaretten raucht. Nikotinentzugserscheinungen sind:

- leichte Erregbarkeit und Ruhelosigkeit,

- Konzentrationsschwäche,

- Angstgefühl (relativ stark ausgeprägt) [37],

- Hungergefühl und Gewichtszunahme,

- Schlafstörungen und Schläfrigkeit,

- heftiges Verlangen nach Zigaretten („Craving“).

Die Entzugserscheinungen sind individuell unterschiedlich stark ausgeprägt und können mehrere Wochen bis Monate anhalten. Zahlreiche Ex-Raucher sind wie Alkoholiker stark gefährdet, weil geringste Anlässe (Besuch einer Gaststätte in Verbindung mit Alkoholgenuss, Zusammentreffen mit Rauchern, plötzlich auftretende Belastungssituationen) der Ausgangspunkt für eine erneute „Raucherkarriere“ sein können. Bei zahlreichen Rauchern sind es aber „nur“ die fehlenden manuellen Beschäftigungen rund um das Zigarettenrauchen [38], die zum erneuten Rauchen führen und damit eine zunächst erfolgreiche Entwöhnungsbehandlung zunichte machen. 


\section{Diagnostik der Tabakabhängigkeit und Indikation zur Behandlung}

Grundsätzlich ist jede Raucherentwöhnung bei einem Patienten sinnvoll vor dem Hintergrund der zahlreichen und bekannten Raucherschäden. Die Raucherentwöhnung hat vier wichtige Indikationen:

1. Verhütung von Erkrankungen aller Art,

2. Behandlung bei Erkrankungen, die mit dem Rauchen assoziiert sind, so z.B. bei einer chronisch-obstruktiven Lungenerkrankung (COPD), Ulcus ventriculi, Hypertonie, koronaren Herzkrankheit, peripheren arteriellen Verschlusskrankheit (PAVK), Hypercholesterolämien, Diabetes mellitus usw.

3. Teil der Rehabilitation nach Erkrankungen, die mit dem Rauchen verknüpft sind (z.B. nach einem durchgemachten Myokardinfarkt, Apoplexie, Amputation einer Extremität) und

4. Schutz der Nichtraucher.

Zur zusammenfassenden Darstellung der Studien zur nicht medikamentösen und medikamentösen Therapie vgl. Haustein [2,39].

\section{Diagnostik}

Neben einer allgemeinen anamnestischen Erhebung sind gezielt Begleiterkrankungen zu erfragen, die als Folge eines jahr(zehnt)elangen Tabakkonsums bedeutsam sind. Für die Bestimmung der Nikotinabhängigkeit ist der Fagerström-Test anzuwenden. Der Grad der Abhängigkeit ist u.a. von der Zahl der täglich gerauchten Zigaretten, aber auch von der Zahl der inhalierten Züge sowie der Inhalationstiefe abhängig. Im Einzelnen sind die in Tab. 1 zusammengefassten Punkte zu erfragen bzw. zu berücksichtigen [40].

\section{Therapieziel}

Die Behandlung des Rauchers sollte in einer vollständigen Entwöhnung bestehen, wobei der plötzliche Rauchstopp angezeigt ist. Dieses Ziel erreichen ohne jede ärztliche Interven- tion allein aus dem eigenen Willen heraus schätzungsweise 10-20\% der Raucher. Dabei ist die Anzahl der täglich konsumierten Zigaretten nicht entscheidend. Diese (abrupte) Rauchstopp-Methode kann mit psychologischer Unterstützung, erfolgversprechender aber mit medikamentöser Unterstützung, erreicht werden. In der Zwischenzeit ist erkannt, dass zahlreiche Raucher aufgrund der starken Abhängigkeit und/oder einer erheblichen Gewohnheitsbildung nicht in der Lage sind, das Rauchen vollständig aufzugeben, so dass eine „Reduktionsbehandlung“ oder partielle Entwöhnung („harm reduction") die dann mögliche Methode sein muss. Dabei sollte erreicht werden, dass der Raucher, zugleich Risikopatient, mit medikamentöser Unterstützung seinen täglichen Konsum auf $<10$ Zigaretten senkt [39].

\section{Medikamentöse Behandlung mit Nikotinpräparaten}

Die Nikotin-Behandlung wurde in den vergangenen 10 Jahren an nahezu 30 Millionen Rauchern angewandt und an mehr als 34800 Rauchern in mehr als 188 Studien erprobt und mehreren Metaanalysen fixiert [41-43]. Für die Evaluierung der Nikotinbehandlung wurden von der Cochrane-Gruppe 108 plazebokontrollierte, prospektive Doppelblindstudien verwertet [44]. Diese Studien waren mit harten Kriterien (Messung der Nikotin-Plasmaspiegel, CO-Messungen etc.) versehen. Damit kann die Behandlung des abhängigen Rauchers mit Nikotin-Präparaten (Pflaster, Kaugummi, Nasalspray, Sublingual-Tabletten, in einigen Ländern auch Inhaler) bedenkenlos durchgeführt werden (Studien vgl. Tab. 2). Raucher mit einer starken „physischen“ Abhängigkeit haben die größten Vorteile von der Nikotin-Behandlung.

\section{Bewertung der Nikotinpräparate}

Wie aus den in Tab. 2 zusammengefassten Ergebnissen zu ersehen ist, sind alle Nikotin-Präparate für die Raucherentwöhnung geeignet: Der 4-mg-Kaugummi ergab höhere Erfolge als die 2-mg-Form jedoch nur bei stark abhängigen Rauchern (Tab. 2) [44-48], weshalb der 2-mg-Kaugummi besser durch die 4-mg-Form ersetzt werden sollte. Bisher wird in den Fachinformationen immer die alleinige Gabe

Tab. 1 Diagnostische Gesichtspunkte bei der Raucherberatung (vgl. [40])

1. Tageskonsum an Zigaretten (regelmäßiger oder unregelmäßiger Konsum)

2. Zigarettenmarke (kann anschließend klassifiziert werden in leicht - mittel - stark)

3. CO-Gehalt der Exspirationsluft (Tageszeit der Messung)

4. Fagerström-Test für die Nikotinabhängigkeit

5. Befragung, ob dissonanter (verabscheut das Rauchen, ist ohne ärztliche Hilfe nicht zu entwöhnen: Nicotine Pre Abstinence Syndrom) oder konsonanter Raucher (entwöhnungsunwillig)

6. Teer-Expositionswert (TEW): korreliert mit dem Risiko eines Bronchialkarzinoms); hängt ab von der Anzahl der Raucherjahre, der täglichen Zigarettenmenge und dem Teergehalt der Zigarettensorte ( $<15 \mathrm{mg}, 15-24 \mathrm{mg}$ oder $>24 \mathrm{mg}$ ).

7. Kohlehydrat-Abhängigkeit (kann bei abhängigen Rauchern zusätzlich auftreten)

8. Größe und Körpergewicht (Berechnung des Bodymass-Index: nachfolgende Gewichtszunahme!)

9. Bisherige Erfahrungen des Rauchers mit Entwöhnungsversuchen (Anzahl der Erfolge/Misserfolge)

10. Auftreten nächtlicher Schlafstörungen und Rauchen während der Unterbrechung des Schlafs (Nocturnal Sleep Disturbing Nicotine Craving: NSDNC)

11. Zusätzlich bestehende Herzkreislauf- oder Pulmonalerkrankungen (bereits durchgemachte Angina-pectoris-Anfälle, Myokardinfarkte, Hypertonie, chronisch-obstruktive Lungenerkrankung, Bronchialkarzinome etc.), gleichzeitig bestehende Psychosen oder Depressionen (können die Entwöhnungsbehandlung komplizieren)

12. Zusätzlich eingenommene Arzneimittel (Neuroleptika, Clozapin, Antidepressiva, Antihypertonika, Lipidhemmer usw.) 


\begin{tabular}{lll}
\hline Medikamentöse Verfahren & Odds Ratio & Bewertung \\
\hline Nikotinpräparate (gesamt) ${ }^{*}$ & $1,73(1,60-1,82)$ & $\uparrow \uparrow$ \\
Kaugummi & $1,63(1,49-1,79)$ & $\uparrow \uparrow$ \\
2 versus 4 mg Kaugummi & $2,67(1,69-4,22)$ & $\uparrow \uparrow$ \\
Pflaster & $1,73(1,56-1,93)$ & $\uparrow \uparrow$ \\
Nasalspray & $2,27(1,61-3,20)$ & $\uparrow \uparrow$ \\
Inhaler & $2,08(1,43-3,04)$ & $\uparrow \uparrow$ \\
Sublingualtablette & $1,73(1,07-2,80)$ & $\uparrow \uparrow$ \\
Bupropion & 2,30 & $\uparrow \uparrow$ \\
mit Nikotin kombiniert & 3,0 & $\uparrow$ \\
\hline
\end{tabular}

Tab. 2 Bewertung medikamentöser Therapieverfahren für die Raucherentwöhnung [44]

\footnotetext{
* Die zusätzliche Beratung steigert die Wirksamkeit.

${ }^{\uparrow \uparrow}$ Aussage (z. B. zur Wirksamkeit) wird gestützt durch mehrere adäquate, valide klinische Studien (z. B. randomisierte klinische Studie) bzw. durch eine oder mehrere valide Metaanalysen oder systematische Reviews. Positive Aussage gut belegt.

$\uparrow$ Aussage (z. B. zur Wirksamkeit) wird gestützt durch zumindest eine adäquate, valide klinische Studie (z. B. randomisierte klinische Studie). Positive Aussage belegt.
}

eines Ersatzpräparates empfohlen. Aus einigen Studien [49-52] geht aber hervor, dass die kombinierte Gabe z. B. von Pflaster plus Kaugummi oder Pflaster plus Nasalspray bei den schwereren Formen der Abhängigkeit größere Erfolge zeigt als die Gabe einer Applikationsform.

Eine zusätzliche individuelle Beratung des Patienten fördert die Erfolgsquote, auch wenn das aus den Studien nicht eindeutig hervorgeht. Die 8-wöchige Behandlung mit Nikotin-Ersatzprodukten war gleich gut wirksam wie längere Behandlungszeiten [53]. Es bestand kein Wirksamkeitsunterschied bei täglicher Verwendung der Pflaster über 16 oder 24 Stunden [53-55].

\section{Behandlung und Dosierung}

Die Behandlung kann mit Nikotinpräparaten über 2-3 Monate geführt werden. Pflaster geben bis zu 1,5 mg Nikotin pro Stunde über die Haut ab. Ob zu einem späteren Zeitpunkt der Behandlung auch Pflaster mit geringeren Stärken (1 bzw. $0,5 \mathrm{mg} /$ Stunde) genutzt werden, ist optional. Kaugummis mit einer Stärke von $4 \mathrm{mg}$ können in einer Zahl bis zu 16 Stück pro Tag eingesetzt werden. Die erfolgreiche und für den Patienten weitgehend symptomlose Anwendung von NikotinKaugummis hängt vom langsamen Gebrauch über 30 Minuten ab. Anstelle des Kaugummis kann künftig auch eine Sublingualtablette zu $2 \mathrm{mg}$ eingesetzt werden (Zahnprothesenträger). Der Nasalspray setzt pro Hub $0,5 \mathrm{mg}$ frei. Es wird je ein Hub pro Nasenloch empfohlen (entspricht $1 \mathrm{mg}$ Nikotin). Es werden 2, maximal 3 Dosen pro Stunde empfohlen (vgl. Tab. 3).

Bei der ärztlichen Führung des Ex-Rauchers muss abgeschätzt werden, wie weit die „Craving“-Effekte nach dem Rauchstopp mit der Zeit zurückgehen, um die Ersatzbehandlung nicht zu zeitig abzusetzen oder die Nikotinzufuhr zu schnell zu reduzieren. Wie aus den in Abb. 1 zusammengefassten Daten ersichtlich, erreicht kein Nikotinpräparat die mit dem Zigarettenrauchen erzielten Plasmaspiegel, weil auch die Nikotinliberation aus Nikotinpräparaten verzögerter als aus der Zigarette erfolgt [56], am schnellsten noch aus dem Nasalspray. Damit wird verständlich, dass sich die täglich ver-

Tab. 3 Praktisches Vorgehen für die Raucherentwöhnung mit Nikotinpräparaten $[2,39]$

Grad der Abhängigkeit und Möglichkeit der Nikotinsubstitution

Sehr starke Abhängigkeit (FTND ${ }^{1} \geq 7$; >25 bis 40 Zigaretten/d; CO²: >30-45 ppm): 1 Nikotin-Pflaster (vgl. Stufe 2)

plus 10-12 Nikotinkaugummis à 4 mg/d plus Nikotin-Nasalspray (vgl. Stufe 3) bedarfsweise, bis „Craving“-Effekte nachlassen; Behandlung über 2-4 Wochen, dann Wegnahme eines Präparates (Kaugummi), später Reduktion der Pflastermenge, Nasalspray für „Craving“-Effekte bis zu einem halben Jahr belassen.

Starke Abhängigkeit (FTND 25; 15-25 Zigaretten/d; CO: 15-35 ppm): 1 Nikotin-Pflaster (vgl. Stufe 2) plus

6-12 Nikotin-Kaugummis/d je nach Rauchverlangen oder Nikotin-Nasalspray ( $10 \mathrm{mg} / \mathrm{ml}$ : je 1 Hub à $0,5 \mathrm{mg}$ je Nasenloch bei jedem Rauchverlangen; maximal: zwei Applikationen stündlich); Behandlung über 3-6 Wochen fortführen, dann allmählich angepasste Dosisreduktion; Versorgung des Ex-Rauchers mit einem Präparat (Kaugummi oder Nasalspray) für „Craving“-Effekte für die darauf folgenden Monate.

Geringere Abhängigkeit (FTND <3; < 15 Zigaretten/d; CO: 10-20 ppm): anfangs bis zu 12 Nikotin-Kaugummis zu 4 mg oder 1 Nikotin-Pflaster (transdermale Abgabe $1,5 \mathrm{mg} / \mathrm{h}$ ), nur in den ersten Behandlungstagen zusätzlich einige Nikotin-Kaugummis à 4 mg; Behandlung je nach Befinden des Ex-Rauchers über 2-3 Wochen fortsetzen, dann angepasste Dosisreduktion.

Ärztliches Gespräch mit Aufklärung des Rauchers über die gesundheitlichen Schäden des Rauchens und die eigene Situation aufgrund des individuellen Befundes.

\footnotetext{
1 Eine innere Korrelation zwischen dem FTDN und den CO-Werten ist nicht belegt. Vielmehr sind beide Werte nur grob miteinander verbunden.

2 Die gemessenen CO-Werte werden durch den Zeitpunkt des Rauchens beeinflusst. Sicherlich ist ein zeitlicher Abstand von 30 Minuten nach der letzten Zigarette bis zur Messung einzuhalten.
} 


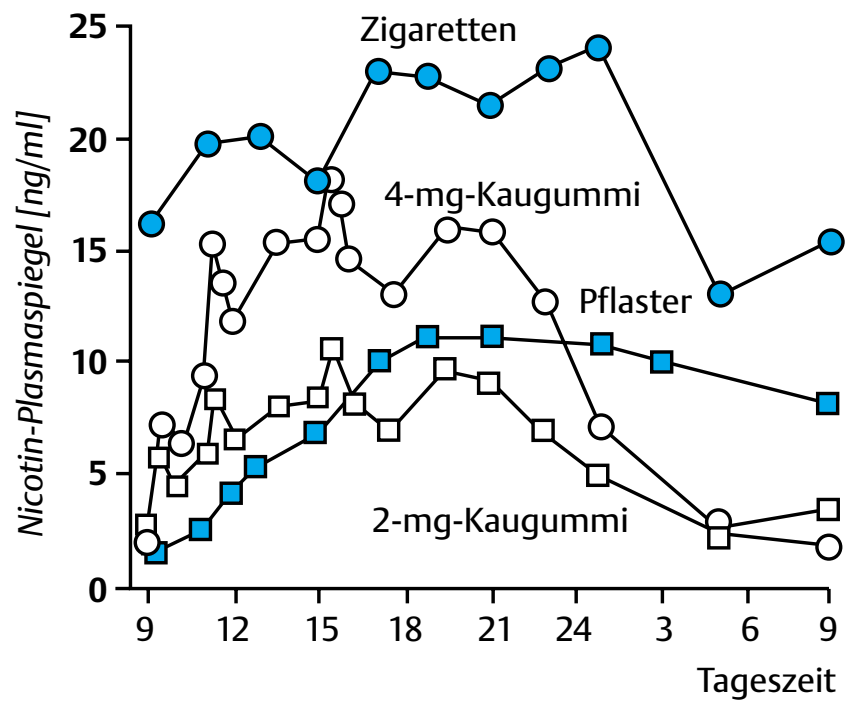

Abb. 1 Nikotin-Plasmaspiegel unter verschiedenen Nikotin-Zubereitungen [78]. Wie aus den in der Abbildung dargestellten Plasmaspiegeln hervorgeht, erreicht keines der Nikotin-Produkte die durch das Zigarettenrauchen erzielten Plasmaspiegel, ebenfalls nicht die schnelle Anflutung.

abreichten Nikotindosen nach dem Grad der Abhängigkeit und der Anzahl der täglich gerauchten Zigaretten richten muss (vgl. Tab. 3).

Insgesamt ist die Nikotin-Ersatztherapie ein (das) geeignete(s) Instrument für die Raucherentwöhnung des abhängigen Rauchers. Die Effektivität der Entwöhnung wird auf das 2- bis 3fache gesteigert, wenn die Raucher zur Entwöhnung bereit sind. Pflaster sind zwar leichter zu handhaben als Kaugummi oder Nasalspray, jedoch können sie in geringerem Maße als insbesondere Nasalspray „Craving“-Effekte unterdrücken. Obwohl nur wenige Studien zur kombinierten Anwendung von 2 verschiedenen Nikotin-Formen (Pflaster + Kaugummi oder Nasalspray bzw. Kaugummi plus Nasalspray) vorliegen, ist die Unterdrückung des plötzlich aufkommenden Rauchwunsches bei zahlreichen entwöhnungswilligen (dissonanten) Ex-Rauchern der entscheidende Punkt für das Versagen bei der einmal begonnenen Entwöhnungsbehandlung. Die ärztliche Führung ist während einer Entwöhnung trotz gegenteiliger Meinungen wichtig [44].

Eigene Erfahrungen bei der Entwöhnung von mehreren hundert Rauchern (Tab.3) sind nicht ohne weiteres zu schematisieren, weil sich zwar der Grad der Abhängigkeit abschätzen lässt, aber durch die Persönlichkeitsstruktur einschließlich des eigenen Willens so determiniert wird, dass der Therapeut immer wieder mit Überraschungen $\mathrm{zu}$ rechnen hat.

Ein wichtiger Punkt ist die eindringliche Warnung an den Patienten, während der Einnahme von Nikotinpräparaten das Zigarettenrauchen zu unterlassen. Der Genuss von 1-2 Zigaretten täglich ist zwar der Entwöhnungsbehandlung abträglich, wenn es sich nicht um das reduzierte Rauchen handelt, wird aber ansonsten vom Patienten toleriert.

\section{Unerwünschte Wirkungen von Nikotin-Präparaten}

Die unerwünschten Wirkungen (UAW) der Nikotin-Präparate sind in Tab. 4 zusammengestellt [39]. Juckreiz oder Brennen auf der Haut unter dem Pflaster treten noch am häufigsten auf. Patienten mit koronarer Herzkrankheit tolerieren Niko-

\begin{tabular}{|c|c|c|}
\hline Applikationsform & Unerwünschte Wirkungen & Häufigkeit \\
\hline \multirow[t]{3}{*}{ Pflaster $^{1}$} & $\begin{array}{l}\text { Lokale Reaktionen: lokale Reaktionen, insbesondere } \\
\text { Hautrötungen }(7,3) \text {, allergische Reaktionen (6-mal), } \\
\text { schwere Lokalreaktionen bzw. mögliche systemische } \\
\text { Überempfindlichkeitsreaktionen (9-mal), } \\
\text { Kontaktekzeme, Hautjucken, Brennen auf der Haut }{ }^{\#}\end{array}$ & $\begin{array}{l}{ }^{\circ} 2,3 \% \\
{ }^{*} \text { oft initial }\end{array}$ \\
\hline & $\begin{array}{l}\text { ZNS: Übelkeit (3,5), anormale Träume }(3,2) \text {, } \\
\text { Schlaflosigkeit }{ }^{~}(3,0) \text {, Schwindel }(2,9), \text { Kopfschmerz }^{\circ} \\
\text { (2,7); Schwindel }{ }^{\circ}(7-\text {-mal), Verwirrung (6-mal), } \\
\text { Erbrechen (6-mal), Krämpfe (5 Fälle) }\end{array}$ & ${ }^{\circ}>1 \%$ \\
\hline & $\begin{array}{l}\text { Kardiovaskuläre Reaktionen: Myokardinfarkt (35-mal) } \\
\text { Brustschmerzen (9-mal), Hirndurchblutungsstörungen } \\
\text { (8-mal), Bluthochdruck (5-mal), Tachykardie (4-mal), } \\
\text { Angina pectoris (3-mal), Herzstillstand (3-mal). } \\
\text { Sonstige: Suizid (einmal }{ }^{2} \text { ) }\end{array}$ & $<0,01 \%$ \\
\hline \multirow[t]{3}{*}{ Kaugummi } & $\begin{array}{l}\text { Lokale Reaktionen: Entzündungen im } \\
\text { Schleimhautbereichs des Mundes, Gingivablutungen; } \\
\text { Hautreaktionen }^{+}\end{array}$ & ${ }^{+}>0,1 \%$ \\
\hline & ZNS: Kopfschmerz, Schwindel und Schlafstörungen & $>1 \%$ \\
\hline & Kardiovaskuläre Reaktionen: Herzklopfen & $>0,1 \%$ \\
\hline Nasalspray & $\begin{array}{l}\text { Lokale Reaktionen: Rhinitis, Nasenbluten, } \\
\text { tränende Augen }\end{array}$ & $>1 \%$ \\
\hline
\end{tabular}

\footnotetext{
1 Aus einer 3-Jahres-Studie an über 3800 Anwendern entnommen (nur teilweise von Ärzten berichtet; $>$ ein Bericht pro Million verkaufter Pflaster).

2 Mit einem stark erhöhten Nikotin-Plasmaspiegel [57, 62]
}

Tab. 4 Unerwünschte Wirkungen unter einer Nikotintherapie. Angaben in ( ) sind in $\%$ angegeben $[2,39]$ 
tin-Pflaster ohne Einschränkungen [57], wobei [58] bei unsachgemäßem Gebrauch Zeichen einer Überdosierung auftreten können (z.B. durch das Kauen mehrerer Kaugummis gleichzeitig oder durch die Kombination von Kaugummi plus Zigaretten). Im Falle von Nikotin führen erste Symptome einer Überdosierung wie Schwindel und Übelkeit zum vorübergehenden Abbruch der Nikotinzufuhr. Auch Zeichen des Nikotin-Entzugs können als unerwünschte Wirkungen fehlgedeutet werden. Beim Nikotin-Nasalspray stehen kurzfristig lokal irritierende Reaktionen (Niesreflex, laufende Nase, Tränen der Augen, Beißen im Rachen, Nasenbluten), Kribbeln im Kopf und Kopfschmerzen im Vordergrund. Dennoch gewöhnen sich zahlreiche Nutzer innerhalb von wenigen Tagen an den vorübergehend zu nutzenden Spray, weil er am schnellsten die „Craving“-Effekte zu beheben vermag $[59,60]$.

Sehr selten kommt es im Rahmen einer Entwöhnungsbehandlung mit Nikotinpräparaten zu einer Abhängigkeitsreaktion, wobei die Ex-Raucher auf Nikotinpräparate (Nasalspray $>$ Kaugummi) fixiert sind und diese dann monatelang weiterverwenden [2]. Hier sollte der behandelnde Arzt wirksam werden.

\section{Wechselwirkungen während des Rauchstopps}

Es gibt keine systematischen Untersuchungen zu Wechselwirkungen zwischen Nikotin und anderen gleichzeitig verabreichten Arzneimitteln. Die metabolischen Prozesse in der Leber werden vor allem durch die Abbrandprodukte des Tabaks und nicht durch Nikotin stimuliert. Tabakrauch induziert die Cytochrom-P450-Enzyme 1A1, 1A2 sowie 2E1 [61]. Daher werden bei Rauchern verschiedene Arzneimittel beschleunigt abgebaut, was andererseits beim Rauchstopp zur Wirksamkeitssteigerung der betroffenen Arzneimittel bei verzögertem Abbau führen kann [62] wie bei Theophyllin, Imipramin, Haloperidol, Tacrin, Koffein, Phenacetin, Phenylbutazon, Estradiol und Pentazocin [61]. Clozapin verzögert den Nikotinabbau [63].

\section{Kontraindikationen für die Anwendung von Nikotin-Ersatzprodukten}

Aufgrund der teilweise falschen Vorstellungen von den Nikotinwirkungen auf das Herz-Kreislauf-System werden in den Fachinformationen zahlreiche Kontraindikationen angegeben und Warnhinweise ausgesprochen, die einer künftigen Revision bedürfen. Die vasokonstriktorischen Wirkungen beim an Nikotin gewöhnten Raucher sind mehr den inhalierten Produkten und dem $\mathrm{CO}$ als dem Nikotin zuzuschreiben [64]. Das trifft möglicherweise auch für die Nikotin-Anwendung während der Schwangerschaft zu [65]. Ansonsten werden als Kontraindikationen genannt: frischer Myokardinfarkt, Herzrhythmusstörungen, kürzlich erfolgter apoplektischer Insult, instabile Angina pectoris, chronisch-generalisierte Hauterkrankungen (Psoriasis, chronische Dermatitiden, Urtikaria). Als relative Kontraindikationen gelten bisher die stabile Angina pectoris, hochgradige Hypertonie, zerebrovaskuläre Erkrankungen, Vasospasmen, schwere Herzinsuffizienz, Hyperthyreoidismus, insulinabhängiger Diabetes mellitus, akute Magen-Darm-Ulzerationen sowie schwere Hautirritationen. Für den Kaugummi kommen hinzu: Entzündungen im Mund-Rachen-Ösophagusbereich, Nieren- und Leberschäden und Fruktose-Intoleranz; für den Nasalspray:
Nasenbluten und chronische Nasenerkrankungen. Vorerst sind Nikotin-Ersatzprodukte nicht während der Schwangerschaft und der Stillzeit anzuwenden, obwohl der Nasalspray bei der Gefahr des Weiterrauchens während der Schwangerschaft erwogen wird [65].

\section{Medikamentöse Behandlung mit Bupropion}

Bei dem seit 10 Jahren in den USA therapeutisch genutzten Antidepressivum Bupropion handelt sich um einen relativ schwachen Aufnahmehemmer für Noradrenalin, 5-Hydroxytryptamin und Dopamin. Bekanntlich wirken auch andere Antidepressiva wie Nortriptylin hemmend auf das Rauchverhalten. Der für den Rauchstopp verantwortliche Mechanismus ist noch unklar, könnte aber mit der Wiederaufnahmehemmung der Transmitter zusammenhängen.

In einer Dosisfindungsstudie [66] wurde Bupropion mit Plazebo bei einer 7-wöchigen Studiendauer verglichen. Nach einer halbjährigen Beobachtung lag der Entwöhnungseffekt in der 300-mg-Gruppe über dem der Plazebogruppe (19 vs. $11 \%)$. Fasst man die vorliegenden Studien mit Bupropion [67-70] zusammen, ergibt sich eine Odds Ratio = 2,73 nach einjähriger Behandlung [71] (vgl. Tab. 2). Bupropion war auch bei Rauchern mit einer vorausgegangenen Depression wirksam [72]. Offensichtlich senkt Bupropion die Entzugssymptome und reduziert die nach dem Rauchstopp auftretende Gewichtszunahme [69]. Im Gegensatz zur Raucherentwöhnung mit Nikotinpräparaten, wo ein Rauchstopp unmittelbar mit dem Beginn der Nikotinverabreichung vereinbart wird, kann der mit Bupropion zu behandelnde Raucher unter der täglichen Einnahme von $150 \mathrm{mg}$ vorerst weiterrauchen und in der 2. Behandlungswoche sich einen Tag für den Rauchstopp unter fortgeführter Bupropioneinnahme aussuchen. $\mathrm{Zu}$ diesem Zeitpunkt wird dann die Tagesdosis auf $300 \mathrm{mg}$ erhöht. Die Behandlung wird über 7-9 Wochen durchgeführt [73].

\section{Unerwünschte Wirkungen und Interaktionen}

Etwa $12 \%$ der Patienten klagten über Schlaflosigkeit und etwa $8 \%$ über einen trockenen Mund [69,74]. In zwei Studien $[69,70]$ trat unter Bupropion unverhältnismäßig häufig Schlaflosigkeit auf (42,4 bzw. 47,5 vs. $19,5 \%$ unter Plazebo), ebenso wurden 5 Fälle von schwerer Depression berichtet (Tab.5) [73]. Des Weiteren wurden vereinzelt Fälle von Stevens-Johnson-Syndrom, allergische Hautreaktionen bis zur Anaphylaxie, Tachykardien, Verwirrtheitszustände und apoplektische Insulte beobachtet. Die bisher vornehmlich im Ausland gesammelten Erfahrungen mit Bupropion bedürfen weiterer Studien [75], wenn eine Überlegenheit gegenüber der Nikotinbehandlung nachgewiesen werden soll. Über weitere unerwünschte Wirkungen vgl. $[73,76]$. Meldungen über etwa 35 Todesfälle aus Großbritannien bedürfen einer eingehenden Analyse bezüglich des Kausalzusammenhangs [75].

\section{Kontraindikationen}

Bupropion sollte nicht bei Patienten mit einem Krampfleiden und bei Bulimie eingesetzt werden [75-77]. Durch die Überdosierung von Bupropion ( $\geq 450 \mathrm{mg} / \mathrm{Tag})$ treten gehäuft Krampfanfälle auf (bei 0,4\% der Patienten) [77]. Die gleichzeitige Einnahme von MAO-Hemmern ist nicht gestattet. Ebenso ist Vorsicht geboten bei der gleichzeitigen Einnahme 
von Antipsychotika, Antidepressiva, Theophyllin und systemisch verabreichten Glukokortikoiden. Mögliche Interaktionen generieren sich vor allem aus der durch Bupropion und Hydroxybupropion ausgelösten Hemmung von Cytochrom $\mathrm{P}_{450}$ 2D6. Betroffen sind Stoffe wie Desipramin. Aber auch andere Antidepressiva (Imipramin, Paroxetin), Antipsychotika (Risperidon, Thioridazon), ß-Blocker (Metoprolol) und Klasse1C-Antiarrhythmika (Propafenon, Flecainid) könnten verzögert abgebaut werden [76]. Diese Arzneimittel sind gegebenenfalls in reduzierter Dosis zu verabreichen oder abzusetzen. Andernfalls ist der Behandlungsabbruch zu erwägen [76].

Während der Schwangerschaft ist Bupropion nur unter sehr strenger Indikationsstellung $\mathrm{zu}$ verwenden. Bupropion und seine Metabolite gehen in die Muttermilch über. Damit besteht die Gefahr der Auslösung von Krämpfen beim Säugling; ein Absetzen der Medikation oder ein Abstillen ist zu überdenken [76].

Insgesamt ist Bupropion aufgrund einer schlechteren NutzenRisiko-Relation als Mittel der 2. Wahl anzuwenden.

\section{Literatur}

${ }^{1}$ Statistisches Bundesamt. Voraussichtliche Straßenverkehrsunfallentwicklung 2000: 3\% weniger Getötete und 4\% weniger Verletzte. Pressemitteilung 21.12.2000

${ }^{2}$ Haustein KO. Tabakabhängigkeit: Gesundheitliche Schäden durch das Rauchen. Deutscher Ärzte-Verlag. Köln, 2001

${ }^{3}$ Peto R, Lopez AD, Boreham J, Thun M, Heath C, Doll R. Mortality from smoking worldwide. Br Med Bull 1996; 52: 12-21 (1)

${ }^{4}$ Haustein KO. Rauchen: Gesundheitswesen und Politik im Wechselspiel. Z ärztl Fortbild Qual sich 1999; 93: 355-361

${ }^{5}$ Benowitz NL, Jaffe JH. Drug addiction an drug abuse: Nicotine and tobacco. In: Gilman AG, Goodman AS, Rall TW, Murrad (Hrsg). Goodman and Gilman's The Pharmacological Basis of Therapeutics. New York: Macmillan, 1985; 4: 554-558

${ }^{6}$ Fagerstrom KO, Kunze M, Schoberberger R, Breslau N, Hughes JR, Hurt RD et al. Nicotine dependence versus smoking prevalence: comparisons among countries and categories of smokers. Tob Control 1996; 5: 52-56 (1)

${ }^{7}$ DSM-IV. American psychiatric association: Diagnostic and Statistical manual of Mental Disorder, ed. 4th. Washington: American Psychatric Association, 1994

${ }^{8}$ Clarke PB. Tobacco smoking, genes, and dopamine. Lancet 1998; 352: 84-85 (9122)

${ }^{9}$ Clarke PB. Dopaminergic mechanisms in the locomotor stimulant effects of nicotine. Biochem Pharmacol 1990; 40: 1427-1432 (7)

${ }^{10}$ Benwell ME, Balfour DJ, Lucchi HM. Influence of tetrodotoxin and calcium on changes in extracellular dopamine levels evoked by systemic nicotine. Psychopharmacology (Berl) 1993; 112: 467-474 (4)

${ }^{11}$ Nisell M, Nomikos GG, Svensson TH. Systemic nicotine-induced dopamine release in the rat nucleus accumbens is regulated by nicotinic receptors in the ventral tegmental area. Synapse 1994; 16: 36-44 (1)

${ }^{12}$ Balfour DJ, Birrell CE, Moran RJ, Benwell ME. Effects of acute D-CPPene on mesoaccumbens dopamine responses to nicotine in the rat. Eur J Pharmacol 1996; 316: 153-156 (2-3)

${ }^{13}$ Shoaib M, Benwell ME, Akbar MT, Stolerman IP, Balfour DJ. Behavioural and neurochemical adaptations to nicotine in rats: influence of NMDA antagonists. Br J Pharmacol 1994; 111: 1073-1080 (4)
${ }^{14}$ Wise RA, Bozarth MA. A psychomotor stimulant theory of addiction. Psychol Rev 1987; 94: 469-492 (4)

${ }^{15}$ Wonnacott S, Irons J, Rapier C, Thorne B, Lunt GG. Presynaptic modulation of transmitter release by nicotinic receptors. Prog Brain Res 1989; 79: 157-163

${ }^{16}$ Benwell ME, Balfour DJ. Regional variation in the effects of nicotine on catecholamine overflow in rat brain. Eur J Pharmacol 1997; 325: 13-20 (1)

${ }^{17}$ Benwell ME, Balfour DJK. Effects of nicotine administration and its withdrawal an plasma corticosterone and brain 5-hydroxinidoles. Psachopharmacology 1979; 4: 7-11

18 Benwell ME, Balfour DJK. The effects of nicotine administration and 5-HT uptake and biosynthesis in rat brain. Eur J Pharmacol 1982; 6: 71-77

${ }^{19}$ Brioni JD, O’Neill AB, Kim DJ, Buckley MJ, Decker MW, Arneric SP. Anxiolytic-like effects of the novel cholinergic channel activator ABT-418. J Pharmacol Exp Ther 1994; 271: 353-361 (1)

${ }^{20}$ Costall B, Kelly ME, Naylor RJ, Onaivi ES. The actions of nicotine and cocaine in a mouse model of anxiety. Pharmacol Biochem Behav 1989; 33: 197-203 (1)

${ }^{21}$ Breslau N, Kilbey MM, Andreski P. Nicotine dependence and major depression. New evidence from a prospective investigation. Arch Gen Psychiatry 1993; 50: 31-35 (1)

22 Bailey SL, Ennett ST, Ringwalt CL. Potential mediators, moderators, or independent effects in the relationship between parents' former and current cigarette use and their children's cigarette use. Addict Behav 1993; 18: 601-621 (6)

${ }^{23}$ Koopmans JR, van Doornen LJ, Boomsma DI. Association between alcohol use and smoking in adolescent and young adult twins: a bivariate genetic analysis. Alcohol Clin Exp Res 1997; 21: 537-546 (3)

${ }^{24}$ Hannah MC, Hopper JL, Mathews JD. Twin concordance for a binary trait. II. Nested analysis of ever-smoking and ex-smoking traits and unnested analysis of a „committed-smoking“ trait. Am J Hum Genet 1985; 37: 153-165 (1)

${ }^{25}$ Heath AC, Madden PA, Slutske WS, Martin NG. Personality and the inheritance of smoking behavior: a genetic perspective. Behav Genet 1995; 25: 103-117 (2)

${ }^{26}$ Pianezza ML, Sellers EM, Tyndale RF. Nicotine metabolism defect reduces smoking. Nature 1998; 393: 750 (6687)

27 Oscarson M, Gullsten H, Rautio A, Bernal ML, Sinues B, Dahl ML et al. Genotyping of human cytochrome P450 2A6 (CYP2A6), a nicotine C-oxidase. FEBS Lett 1998; 438: 201-205 (3)

${ }^{28}$ Spitz MR, Shi H, Yang F, Hudmon KS, Jiang H, Chamberlain RM et al. Case-control study of the D2 dopamine receptor gene and smoking status in lung cancer patients. J Natl Cancer Inst 1998; 90: 358-363 (5)

${ }^{29}$ Blum K, Noble EP, Sheridan PJ, Montgomery A, Ritchie T, Jagadeeswaran $\mathrm{P}$ et al. Allelic association of human dopamine D2 receptor gene in alcoholism. JAMA 1990; 263: 2055-2060 (15)

${ }^{30}$ Blum K, Noble EP, Sheridan PJ, Montgomery A, Ritchie T, Ozkaragoz $\mathrm{T}$ et al. Genetic predisposition in alcoholism: association of the D2 dopamine receptor TaqI B1 RFLP with severe alcoholics. Alcohol 1993; 10: 59-67 (1)

${ }^{31}$ Noble EP. The D2 dopamine receptor gene: a review of association studies in alcoholism. Behav Genet 1993; 23: 119-129 (2)

32 Noble EP, Blum K, Khalsa ME, Ritchie T, Montgomery A, Wood $\mathrm{RC}$ et al. Allelic association of the D2 dopamine receptor gene with cocaine dependence. Drug Alcohol Depend 1993; 33 : 271-285 (3)

${ }^{33}$ Comings DE, Ferry L, Bradshaw-Robinson S, Burchette R, Chiu C, Muhleman D. The dopamine D2 receptor (DRD2) gene: a genetic risk factor in smoking. Pharmacogenetics 1996; 6: 73-79 (1) 
${ }^{34}$ Lerman C, Caporaso NE, Audrain J, Main D, Bowman ED, Lockshin B et al. Evidence suggesting the role of specific genetic factors in cigarette smoking. Health Psychol 1999; 18: 14-20 (1)

35 True WR, Xian H, Scherrer JF, Madden PA, Bucholz KK, Heath AC et al. Common genetic vulnerability for nicotine and alcohol dependence in men. Arch Gen Psychiatry 1999; 56: 655-661 (7)

${ }^{36}$ Yates WR, Cadoret RJ, Troughton EP, Stewart M, Giunta TS. Effect of fetal alcohol exposure on adult symptoms of nicotine, alcohol, and drug dependence. Alcohol Clin Exp Res 1998; 22: 914-920 (4)

${ }^{37}$ West R, Hajek P. What happens to anxiety levels on giving up smoking? Am J Psychiatry 1997; 154: 1589-1592 (11)

${ }^{38}$ Haustein KO. Tabakabhängigkeit - Erfahrungen aus dem Raucherberatungszentrum. In: Zerdick J (Hrsg). Vol. 5: Suchtmedizin im Dialog, Deutsche Gesellschaft für Suchtmedizin. Berlin: Verlag für Wissenschaft und Bildung, 2001: 221-231

${ }^{39}$ Haustein KO. Pharmacotherapy of nicotine dependence. Int J Clin Pharmacol Ther 2000; 38: 273-290 (6)

${ }^{40}$ Schoberberger RKM. Nikotinabhängigkeit: Diagnostik und Therapie. Wien, New York: Springer-Verlag, 1999

${ }^{41}$ Fiore MC, Smith SS, Jorenby DE, Baker TB. The effectiveness of the nicotine patch for smoking cessation. A meta-analysis. JAMA 1994; 271: 1940-1947 (24)

${ }^{42}$ Silagy C, Mant D, Fowler G, Lodge M. Meta-analysis on efficacy of nicotine replacement therapies in smoking cessation. Lancet 1994; 343: 139-142 (8890)

${ }^{43}$ Tang JL, Law M, Wald N. How effective is nicotine replacement therapy in helping people to stop smoking? BMJ 1994; 308 : 21-26 (6920)

${ }^{44}$ Silagy C, Mant D, Fowler G, Lancaster T. Nicotine replacement therapy for smoking cessation. Cochrane Database Syst Rev 2000; : CD000146 (3)

${ }^{45}$ Herrera N, Franco R, Herrera L, Partidas A, Rolando R, Fagerstrom KO. Nicotine gum, 2 and $4 \mathrm{mg}$, for nicotine dependence. A double-blind placebo-controlled trial within a behavior modification support program. Chest 1995; 108: 447-451 (2)

${ }^{46}$ Kornitzer M, Kittel F, Dramaix M, Bourdoux P. A double blind study of $2 \mathrm{mg}$ versus $4 \mathrm{mg}$ nicotine-gum in an industrial setting. J Psychosom Res 1987; 31: 171-176 (2)

${ }^{47}$ Tonnesen P, Fryd V, Hansen M, Helsted J, Gunnersen AB, Forchammer $\mathrm{H}$ et al. Two and four mg nicotine chewing gum and group counselling in smoking cessation: an open, randomized, controlled trial with a 22 month follow-up. Addict Behav 1988; 13: 17-27 (1)

${ }^{48}$ Tonnesen P, Fryd V, Hansen M, Helsted J, Gunnersen AB, Forchammer $\mathrm{H}$ et al. Effect of nicotine chewing gum in combination with group counseling on the cessation of smoking. N Engl J Med 1988; 318: 15-18 (1)

${ }^{49}$ Blondal T, Gudmundsson LJ, Olafsdottir I, Gustavsson G, Westin A. Nicotine nasal spray with nicotine patch for smoking cessation: randomised trial with six year follow up. BMJ 1999; 318: 285-288 (7179)

${ }^{50}$ Bohadana A, Nilsson F, Rasmussen T, Martinet Y. Nicotine inhaler and nicotine patch as a combination therapy for smoking cessation: a randomized, double-blind, placebo-controlled trial. Arch Intern Med 2000; 160: 3128-3134 (20)

${ }^{51}$ Kornitzer M, Boutsen M, Dramaix M, Thijs J, Gustavsson G. Combined use of nicotine patch and gum in smoking cessation: a placebo-controlled clinical trial. Prev Med 1995; 24: 41-47 (1)

${ }^{5}$ Puska PK. Combined use of nicotine patch and gum compared with gum alone in smoking cessation: a clinical trial in North Karelia. Tobacco Control 1995; 4: 231-235

${ }^{53}$ Tonnesen P, Paoletti P, Gustavsson G, Russell MA, Saracci R, Gulsvik A et al. Higher dosage nicotine patches increase oneyear smoking cessation rates: results from the European CEASE trial. Collaborative European Anti-Smoking Evaluation. European Respiratory Society. Eur Respir J 1999; 13: 238-246 (2)

${ }^{54}$ Daughton DM, Heatley SA, Prendergast JJ, Causey D, Knowles M, Rolf $\mathrm{CN}$ et al. Effect of transdermal nicotine delivery as an adjunct to low-intervention smoking cessation therapy. A randomized, placebo-controlled, double-blind study. Arch Intern Med 1991; 151: 749-752 (4)

${ }^{55}$ Daughton DM, Fortmann SP, Glover ED, Hatsukami DK, Heatley SA, Lichtenstein E et al. The smoking cessation efficacy of varying doses of nicotine patch delivery systems 4 to 5 years post-quit day. Prev Med 1999; 28: 113-118 (2)

${ }^{56}$ Blondal T. Controlled trial of nicotine polacrilex gum with supportive measures. Arch Intern Med 1989; 149: 1818-1821 (8)

57 Transdermal nicotine for smoking cessation. Six-month results from two multicenter controlled clinical trials. Transdermal Nicotine Study Group. JAMA 1991; 266: 3133-3138 (22)

58 Joseph AM, Antonnucio DO. Lack of efficacy of transdermal nicotine in smoking cessation. N Engl J Med 1999; 341: 1157-1158 (15)

${ }^{59}$ Batra A, Schupp PE, Buchkremer G. Die Behandlung von schwerabhängigen Rauchern mit Nikotinpflaster und Nikotinnasenspray. In: Haustein KO (Hrsg). Rauchen und Nikotin - Aktuelle Beiträge zur Raucherentwöhnung. Nürnberg: Verlag Perfusion GmbH, 1998: 49-58

${ }^{60}$ Blondal T, Franzon M, Westin A. A double-blind randomized trial of nicotine nasal spray as an aid in smoking cessation. Eur Respir J 1997; 10: 1585-1590 (7)

${ }^{61}$ Zevin S, Benowitz NL. Drug interactions with tobacco smoking. An update. Clin Pharmacokinet 1999; 36: 425-438 (6)

${ }^{62}$ Miller LG. Recent developments in the study of the effects of cigarette smoking on clinical pharmacokinetics and clinical pharmacodynamics. Clin Pharmacokinet 1989; 17: 90-108 (2)

${ }^{63}$ Skogh E, Bengtsson F, Nordin C. Could discontinuing smoking be hazardous for patients administered clozapine medication? A case report. Ther Drug Monit 1999; 21: 580-582 (5)

${ }^{64}$ Haustein KO. Smoking tobacco, microcirculatory changes and the role of nicotine. Int J Clin Pharmacol Ther 1999; 37: 76-85 (2)

${ }^{65}$ Haustein KO. Cigarette smoking, nicotine and pregnancy. Int J Clin Pharmacol Ther 1999; 37: 417-427 (9)

${ }^{66}$ Hurt RD, Offord KP, Croghan IT, Gomez-Dahl L, Kottke TE, Morse RM et al. Mortality following inpatient addictions treatment. Role of tobacco use in a community-based cohort. JAMA 1996; 275: 1097-1103 (14)

${ }^{67}$ Ferry LH BR. Efficacy of bupropion for smoking cessation in non depressed smokers [Abstract]. Addict Dis 1994; 13: 249

${ }^{68}$ Ferry LH RASP. Enhancement of smoking cessation using the antidepressant bupropion hydrochloride [Abstract 2670]. Circulation 1992; 86: I-671 (Suppl 1)

${ }^{69}$ Hurt RD, Sachs DP, Glover ED, Offord KP, Johnston JA, Dale LC et al. A comparison of sustained-release bupropion and placebo for smoking cessation. N Engl J Med 1997; 337: 1195-1202 (17)

${ }^{70}$ Jorenby DE, Leischow SJ, Nides MA, Rennard SI, Johnston JA, Hughes AR et al. A controlled trial of sustained-release bupropion, a nicotine patch, or both for smoking cessation. N Engl J Med 1999; 340: 685-691 (9)

${ }^{71}$ Hughes JR, Stead LF, Lancaster T. Anxiolytics and antidepressants for smoking cessation (Cochrane Review). The Cochrane Library, editor. Oxford: Issue 3, 2000, 2000

${ }^{72}$ Hayford KE, Patten CA, Rummans TA, Schroeder DR, Offord KP, Croghan IT et al. Efficacy of bupropion for smoking cessation in smokers with a former history of major depression or alcoholism. Br J Psychiatry 1999; 174: 173-178 
${ }^{73}$ Haustein KO. Antidepressivum kontra Nicotin: Neue Methoden der Raucherentwöhnung. Münch med Wschr/Fortschr Med 2000; 142: 988-990

${ }^{74}$ Goldstein MG. Bupropion sustained release and smoking cessation. J Clin Psychiatry 1998; 59 Suppl 4: 66-72

75 anonym . Bupropion: update. Canad ADR Newsletter 2000; 10: 3-7

${ }^{76}$ anonym. Fachinformation zu Zyban R: Darstellung der Eigenschaften von Bupropion (Glaxo-Wellcome), Stand Juni 2000. 2000. 30.6.2000.

${ }^{77}$ Dunner DL, Zisook S, Billow AA, Batey SR, Johnston JA, Ascher JA. A prospective safety surveillance study for bupropion sustainedrelease in the treatment of depression. J Clin Psychiatry 1998; 59: 366-373 (7)

${ }^{78}$ Tang JL, Law M, Wald N. How effective is nicotine replacement therapy in helping people to stop smoking? BMJ 1994; 308: 21-26 (6920)
Prof. Dr. med. Knut-Olaf Haustein

Institut für Nikotinforschung und Raucherentwöhnung Johannesstraße 85-87 99084 Erfurt

E-mail: haustein@inr-online.de 